\title{
Validation of a Device for Clinical Measurement of Frontal Tibiofemoral Alignment
}

\author{
C. van Giersbergen ${ }^{1}$, J. Bellemans ${ }^{2}$, P. Moens ${ }^{2}$, A. J. F. Hosman ${ }^{1}$ \\ ${ }^{1}$ Orthopedics, University Medical Center Sint Radboud, Nijmegen, The Netherlands; ${ }^{2}$ Orthopaedic Research, University Hospital \\ Louvain, Clinic Pellenberg, Leuven, Belgium. \\ Email: chantalvangiersbergen@student.ru.nl
}

Received May $4^{\text {th }}, 2012$; revised June $18^{\text {th }}, 2012$; accepted July $21^{\text {st }}, 2012$

\begin{abstract}
Study Design: A prospective instrumental study design. Objectives: In this study, the validity of a device was investigated. Background: Frontal tibiofemoral malalignment is a predisposing factor for developing gonarthrosis. In a previous study a repositioning device was developed to clinically measure frontal tibiofemoral alignment. This device appeared to be more accurate than the conventional method. Case Description: This study has examined three types of measurement: conventional method, repositioning device and a bilateral full-leg standing radiograph (Rx). We compared the results of the repositioning device and the conventional method to Rx. To determine the validity of the new repositioning device, $\mathrm{n}=41$ patients under 18 years of age were included in this study. They all underwent a standing $\mathrm{Rx}$ in their history. Outcome: In our study we found a negative correlation between the repositioning device and the already known Rx. Conclusion: The repositioning device was validated, but is not accurate enough to replace Rx. However, physiotherapists can use this device to obtain a more accurate clinical measurement of frontal tibiofemoral alignment.
\end{abstract}

Keywords: Radiographics; Knee Alignment; Radiation

\section{Introduction}

Frontal tibiofemoral alignment (i.e. genu varum, normal or genu valgum) is a clinically important entity as frontal tibiofemoral malalignment is a predisposing factor for developing gonarthrosis [1-5]. Varus or valgus weight bearing of a joint can induce wear and tear in a joint causing gonarthrosis [6,7]. Genu varum can cause medial gonosteoarthritis if major adduction movements occur $[8,9]$. Arthrosis of the knee can give an additional complication: secondary arthrosis of the ankle [10]. In some studies [11,12], a correlation was observed between developing genu varum and an increased sports activity during growth. All these factors suggest it is important to clinically detect and follow up frontal tibiofemoral malalignment of the knee in children with increased sports activity in order to detect early onset of gonarthrosis.

The conventional method of measuring frontal knee alignment appeared to be unreliable, as subjects did not relax their muscles in this position [12]. In the study of Witvrouw et al., a new repositioning device (WRD) was developed to clinically measure frontal knee alignment (Figure 1) [12]. This WRD allows measurement of the intercondylar (IC) distance (distance between both medial condyles) and intermalleolar (IM) distance (distance between both medial malleoli) of the legs.

If this WRD is used, a subject is not able to influence the measurement as the subject cannot actively put their legs together by muscle force. This allows the researchers to obtain a more realistic idea of the frontal knee alignment. To appreciate the clinical use of the WRD its efficacy should be compared with the golden standard (Rx: bilateral full-leg standing radiographs) [6,13-15]. Additionally, when the WRD is efficient enough, unnecessary harmful bilateral full-leg standing radiographs can be prevented among children in the future.

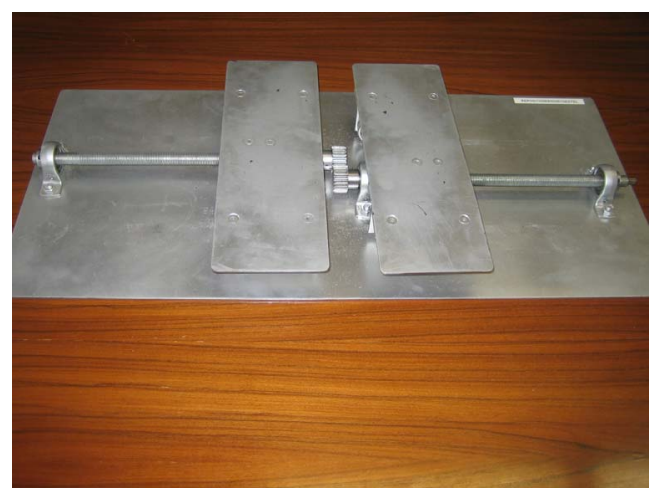

Figure 1. Repositioning device (WRD). 
This study was performed to determine if the WRD is a valid and reproducible method of measuring frontal tiobiofemoral alignment, as compared to the Rx. For this purpose we measured frontal knee alignment in $n=41$ patients under 18 years of age using both techniques and thereby comparing these techniques with each other and with Rx.

Previous literature demonstrated a high intra- and interobserver reliability using these methods $[13,16]$.

\section{Methods}

This study is a comparative experimental instrumental prospective study and was approved by the Medical Ethical Committee in Louvain. All subjects signed the informed consent.

The rights of the subjects were protected.

The equipment was calibrated every measurement.

For all subjects, frontal knee alignment was measured three times using different methods: the conventional method, the WRD and the Rx, where the Rx will function as the control/golden standard.

\subsection{Study Population: Inclusion and Exclusion Criteria}

The study subjects were children under 18 years of age who had taken in their recent history, up until 1 year ago, a bilateral full-leg standing radiograph. The time limit is set on 1 year, because we needed to include the influence of the growth rate of the femur and tibia on the frontal tibiofemoral alignment.

The exclusion criteria: children who were too young to take a standing $\mathrm{Rx}$, mentally ill children who were not able to stand still during the measurements, when standing on the WRD and the toes faced inward while the patellae were faced forward, when both patellae were not in the centre on the $\mathrm{Rx}$, when the patellae faced forward on $\mathrm{Rx}$ and the toes faced inward, when the subject had undergone an operation that could intervene with frontal tibiofemoral alignment after Rx was made.

The subjects with their toes facing inward where excluded due a lack of rotational function on the WRD.

\subsection{Conventional Method}

The measurement of the IC/IM distance is described by asking the subject to stand erect in anatomic position with the medial condyles or malleoli touching and the hips and knees in maximal extension, as described by $\mathrm{J}$. C. Cheng, et al. [17].

When there was rotation in the leg, the patellae did not face forward. To correct this tibial rotation, the feet were rotated inward or outward until the patellae did face forward.

A electronically calliper was used and measures up until 2 decimals precise. The value of the IC/IM distance in millimetres was the result of a duplicate measurement.

\subsection{The WRD (New Method)}

Subjects were asked to take a relaxed position on the device by putting each foot on a different platform (Figure 1). The medial side of the foot must be placed against the inner border of the platform, and heels must be on the same line. This position forces all subjects to point their feet straight forward. This minimizes the rotational effects of the hips. However, when the patellae where not facing forward, the feet were rotated until the patellae did face forward.

A motor moves the platforms at a constant low speed to each other until the condyles and/or malleoli touch [12].

The IC and IM distance in millimetres was measured with an inside calliper using the method described by Arazi et al. [18].

The value for the IC/IM distance was the result of a duplicate measurement. There was agreement that the value of the IC distance was positive and the value of the IM distance was negative. To eliminate negatives in our data, we added +100 to the total sum of the IC/IM distance. When a subject was positioned correctly, but neither the two malleoli nor the condyles touched due to fat among the knee or feet that touched earlier than the malleoli, the IC/IM distance was also measured. The fat among the knee was compressed until the fingertip of the examiner could not fit between the condyles any more. These IC and IM distances measured at the same subject were inverted to an absolute IC/IM distance by subtracting IM distance from IC distance.

The IC/IM distances were compared with the genu varum, normal and genu valgum angles measured by $\mathrm{Rx}$.

\subsection{The Golden Standard/Rx}

Bilateral full-leg standing radiographs were obtained as described by Paley with the subjects standing barefoot and the feet together in the "stand at attention" position while the patellae were oriented forward. The whole pelvis was included in the radiographs and de gonads were always shielded [12,19].

To determine whether there was a genu varum or genu valgum, the hip-knee-ankle (HKA) axis was used as described by Tallroth K. et al. (Figure 2) [10]. The HKA axis was defined as the angle formed by the mechanical femoral axis and the mechanical tibial axis. The HKA axis was expressed as a deviation from $180^{\circ}$ with a negative value for varus-alignment and positive value for valgus-alignment [11]. These angles were measured on the bilateral full-leg standing radiographs. To eliminate negatives in our data, we added +10 to each value. For measurement of $\mathrm{Rx}$ the patellae must be in the centre of 


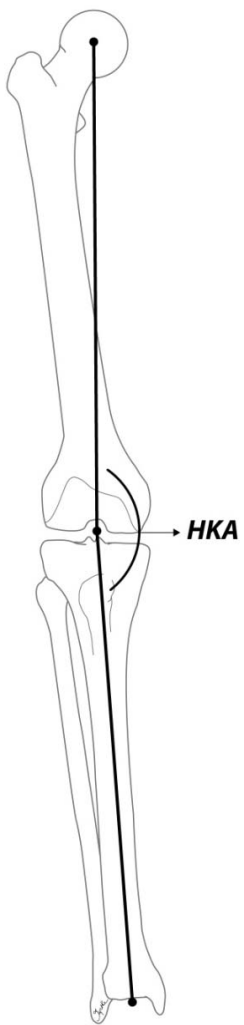

Figure 2. The mechanical axis (HKA axis) of the lower leg is defined as a line from the centre of the femoral head to the centre of the ankle. For a $0^{\circ}$ mechanical axis of the limb, the line should pass through the centre of the knee. In a varus knee, the mechanical axis of the limb passes medially to the centre of the knee. The deviation angle is formed by a line from the centre of the femoral head to the centre of the knee and a line from the centre of the knee to the centre of the ankle [22].

the knee, which results in a minimal additional rotation of the hip [11,19-26]. If the patellae were not in the centre of the knee, Rx data could not be compared to the IC/IM distance. If only one patella was not in the centre of the knee, only the leg with the patella in the centre was included in the data. The value of the angles were in degrees $\left(^{\circ}\right)$. Each value was the result of a duplicate measurement. To combine the right and left HKA axis, both values were added up and divided by two.

The measurement of the body length (in centimetres) was done by a measuring rod along the wall. This was to see whether the body length was correlated to the IC/IM distance. In addition we would like to determine whether the IC/IM distance could be correlated with a certain frontal malalignment of the knee per length.

\subsection{Statistical Analysis}

The resemblance for both methods to Rx was quantified with the "within-subject" standard deviation (also known as the "standard error of the mean" (SEM) in a test-retest study) and an "intra-class" correlation (ICC) (McGraw \& S. P. Wong, 1996).

A Spearman correlation with the absolute difference showed that $p$-values $\leq 0.05$ were considered statistically significant; all tests were 2-tailed.

\section{Results}

The study started on November 16th 2011 and ended at January 11th 2012.

Characteristics of the study population: sex, body length, body-mass-index (BMI), IC/IM distance of the conventional and new method and HKA axis on the bilateral full-leg standing radiographs in degrees.

In total, 41 subjects had signed the informed consent. 2 dropped out because personal reasons, one subject had not taken a bilateral full-leg standing radiograph. In 4 subjects, the bilateral full-leg standing radiograph did not meet the specification of time limit: 1 year.

For both methods, the resemblance with $\mathrm{Rx}$ was visualized with a Bland-Altman plot (Table 1 and Figure 3, Table 2 and Figure 4). This plot shows the difference of the method (compared to $\mathrm{Rx}$ ) as to the mean of the method.

\section{Bland-Altman of Conventional Method vs Rx: Difference vs average}

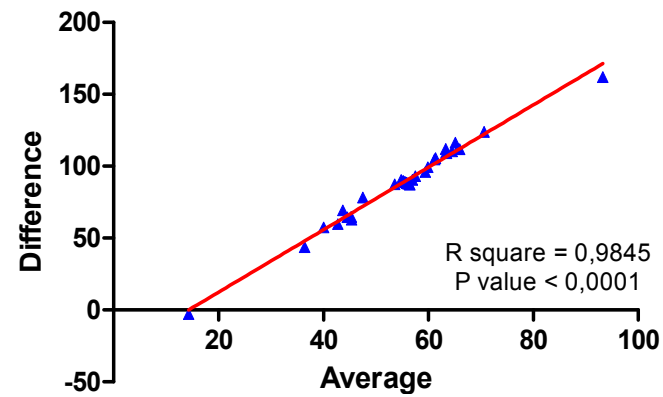

Figure 3. Bland-Altman plot; conventional method versus Rx.

\section{Bland-Altman of New Method vs Rx: Difference vs average}

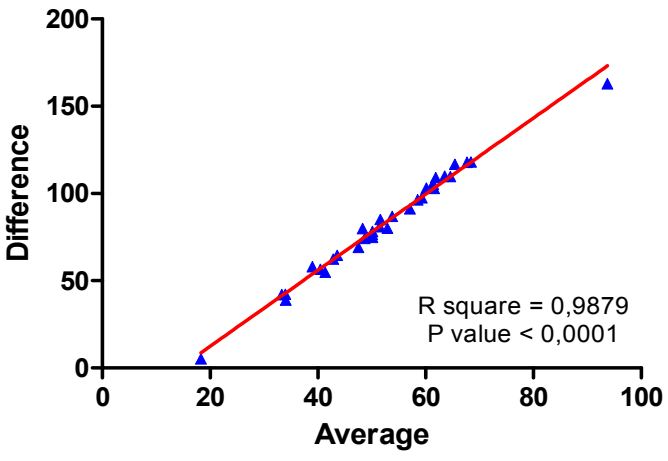

Figure 4. Bland-Altman plot; new method versus Rx. 
Table 1. Bland-Altman bias; conventional method versus Rx.

\begin{tabular}{cc}
\hline Bias & 89.34 \\
\hline Standard deviation of bias & 27.82 \\
$95 \%$ limits of agreement & \\
From & 34.82 \\
To & 143.9 \\
\hline
\end{tabular}

Table 2. Bland-Altman bias; new method versus $R x$.

\begin{tabular}{cc}
\hline Bias & 84.10 \\
\hline Standard deviation of bias & 29.81 \\
$95 \%$ limits of agreement & \\
From & 25.67 \\
To & 142.5 \\
\hline
\end{tabular}

The bias of the conventional method to Rx is 89.34 . The $95 \%$ "limits of agreement" in this plot shows the interval where $95 \%$ of the difference will be.

The $\mathrm{R}^{2}$ of the slope in Figure 3 is 0.9845 . That is a strong correlation between the conventional method and $\mathrm{Rx}$.

The bias of the new method is smaller than the conventional method. Therefore, the new method a better comparison to Rx than the conventional method.

The $\mathrm{R}^{2}$ of the slope in Figure 4 is 0.9879 . That is an even stronger correlation between the new method and $\mathrm{Rx}$ than the conventional method.

The Spearman correlation between the mean and the difference in the Bland-Altman plot shows whether the direction of trend of the difference with Rx depends on the level of measurement.

The Spearman correlation between both methods is 0.77 with a $\mathrm{p}$ value of $7.917238 \mathrm{e}^{-0.09}$. This shows that both measuring methods deliver approximately the same outcome.

To determine if the new method can replace the conventional method is shown in Tables $\mathbf{3}$ and $\mathbf{4}$.

Table 3 states that $60 \%$ (Spearman r) of the result from the conventional method is dependent on the variable we used, which means that $40 \%$ of the true frontal tibiofemoral alignment is depending on variables we cannot measure in this study.

Table 4 states that $70 \%$ (Spearman r) of the result from the new method is dependent on the variable we used. This means that $30 \%$ of the true frontal tibiofemoral alignment dependents on variables we cannot measure in this study. It can be concluded that the new method is better than the conventional method to measure the frontal tiobiofemoral alignment.

The negative values of the Spearman correlation state that with an increasing distance (millimeters) there is a decrease in degrees $\left({ }^{\circ}\right)$ in the angle shown on Rx. This is correct if there were only valgum-alignments of the knee.

\section{Bland-Altman of New Method vs Rx: Difference vs average}

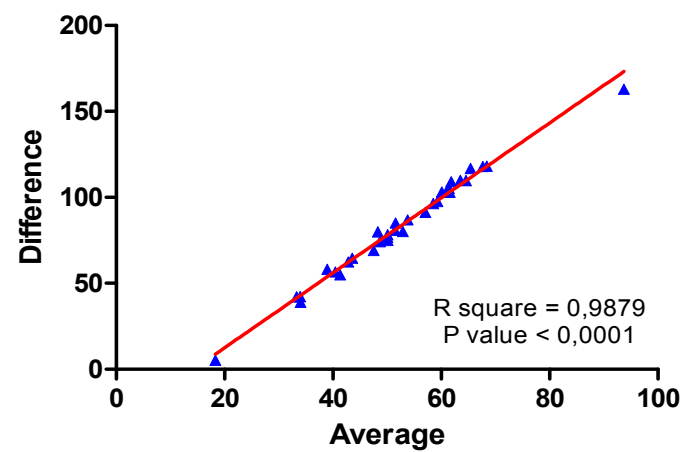

Figure 4. Bland-Altman plot; new method versus Rx.

Intra-Class Correlation of Conventional Method vs Rx

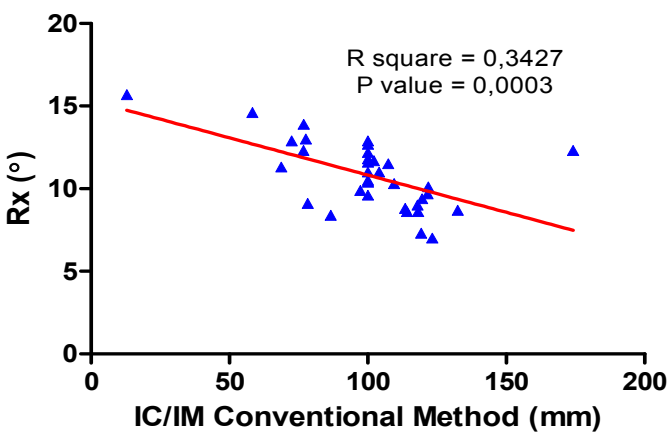

Figure 5. Intra-class correlation for conventional methodcompared to $\mathbf{R x}$.

Table 3. Spearman correlation; conventional method versus Rx.

\begin{tabular}{ll}
\hline & Rx: HKA-angle $\left(^{\circ}\right)$ \\
\hline Spearman $\mathrm{r}$ & -0.6055 \\
$95 \%$ confidence interval & -0.7873 to -0.3268 \\
P value (two-tailed) & 0.0001 \\
$\begin{array}{l}\text { Exact or approximate P value? } \\
\begin{array}{l}\text { Is the correlation significant? } \\
\text { (alpha }=0.05)\end{array}\end{array}$ & Gaussian approximation \\
\hline
\end{tabular}

Table 4. Spearman correlation; new method versus $\mathbf{R x}$.

\begin{tabular}{ll}
\hline & Rx: HKA-angle $\left(^{\circ}\right)$ \\
\hline Spearman $\mathrm{r}$ & -0.6987 \\
$95 \%$ confidence interval & -0.8418 to -0.4638 \\
p value (two-tailed) & $<0.0001$ \\
$\begin{array}{l}\text { Exact or approximate p value? } \\
\begin{array}{l}\text { Is the correlation significant? } \\
\text { (alpha }=0.05)\end{array}\end{array}$ & Ges \\
\hline
\end{tabular}


We can conclude that there were more valgumalignments than varum-alignments of the knee in our data.

The intra-class correlation (ICC) for the conventional method is 0.3427 and for the new method 0.4125 (Figures 5 and 6). We can conclude the new method is better than the conventional method.

The correlation between body length and IC/IM distance is $0.384(\mathrm{p}=0.016)$ for the conventional method and $0.296(\mathrm{p}=0.068)$ for the new method. This states there is a weak correlation between body length and IC/IM distance. Due to this, there is no need to correlate between IC/IM distance and frontal malalignment of the knee per body length.

We also correlated between BMI and IC/IM distance: $0.08519019(\mathrm{p}=0.6265599)$ for the conventional method and $-0.03166375(\mathrm{p}=0.8567073)$ for the new method. We conclude that there is no correlation between BMI and IC/IM distance.

It can also be concluded that the new method cannot replace the bilateral full-leg standing radiographs.

\section{Discussion}

We managed to validate the WRD, but the correlation with $\mathrm{Rx}$ is not high enough to replace Rx. Still, the WRD is better for the clinical measurement of frontal tibiofemoral alignment than the conventional method.

All methods in this study were used in previous studies and considered accurate. To make the measurement better, we added the check of "the finger between the knees" to be sure the medial condyles touched.

We made adjustments to the methods used in previous studies to correct rotation: we rotated the feet until the patellae faced forward. However, we could not correct for external rotation, because the platforms on the WRD could not rotate. That is why some variables that influence frontal tibiofemoral alignment could not be included in this study: ante-version of the hips and tibial endorotation.

\section{Intra-Class Correlation of New Method vs Rx}

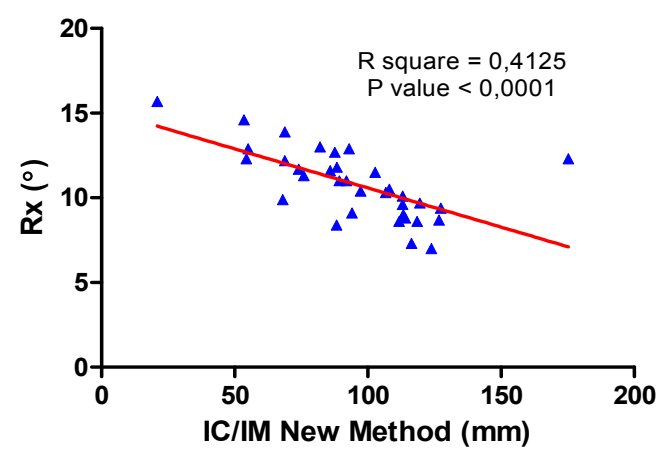

Figure 6. Intra-class correlation for new method compared to $\mathbf{R x}$.
The correlation between IC/IM distance and BMI is 0.08519019 for the conventional method and -0.03166375 for the new method. A shortcoming of this study was that the study population was too small to conclude whether or not a correlation between BMI and IC/IM distance exists. In this study were only 3 out of 41 subjects who had a BMI above 25. In the normal population, approximately 22 percent of all children have a BMI above 25 [27].

The WRD is not good enough to replace Rx, but physiotherapists can use this WRD to obtain a more accurate clinical measurement of frontal tibiofemoral alignment. And therefore determine a better manual therapy for their patients.

\section{Conclusions}

In summary, our study showed a better correlation between the WRD and Rx than the conventional method. However, this correlation is not good enough to replace Rx.

Physiotherapist can use this WRD to obtain a more accurate clinical measurement of frontal tibiofemoral alignment.

\section{Acknowledgements}

The authors thank Myrthe Boymans for her illustration included in this manuscript. The statistical analysis was performed by the Biostatistical Centre of the School of Public Health of the Catholic University Leuven.

\section{REFERENCES}

[1] V. Lun, H. Meeuwisse, P. Stergiou and D. Stefanyshyn, "Relation between Running Injury and Static Lower Limb Alignment in Recreational Runners," British Journal of Sports Medicine, Vol. 38, No. 5, 2004, pp. 576-580. doi:10.1136/bjsm.2003.005488

[2] S. P. Messier, S. E. Davis, W. W. Curl, R. B. Lowery and R. J. Pack, "Etiologic Factors Associated with PF Pain in Runners," Medicine \& Science in Sports \& Exercise, Vol. 23, No. 9, 1991, pp. 1008-1015. doi:10.1249/00005768-199109000-00003

[3] J. E. Tauton, M. B. Ryan, D. B. Clement, D. C. McKenzie, D. R. L. Smith and B. D. Zumbo, "A Retrospective Case-Control Analysis of 2002 Running Injuries," British Journal of Sports Medicine, Vol. 36, No. 2, 2002, pp. 95101. doi:10.1136/bjsm.36.2.95

[4] W. van Mechelen, "Running Injuries. A Review of the Epidemiological Literature," Sports Medicine, Vol. 14, No. 5, 1992, pp. 320-335. doi:10.2165/00007256-199214050-00004

[5] D. Y. Wen, J. C. Puffer and T. P. Schmalzried, "Injuries in Runners: A Prospective Study of Alignment," Clinical Journal of Sport Medicine, Vol. 8, No. 3, 1998, pp. 187194. doi:10.1097/00042752-199807000-00005 
[6] N. Fakhrai, P. Widhalm, C. Chairi, M. Weber, et al., "Automatic Assessment of the Knee Alignment Angle on Full-Limb Radiographics," European Journal of Radiology, Vol. 74, No. 1, 2010, pp. 236-240. doi:10.1016/j.ejrad.2009.02.004

[7] R. Schmitz, S. Shultz and A. Nguyen, "Dinamic Valgus Alignment and Functional Strength in Males and Females during Maturation," Journal of Athletic Training, Vol. 44, No. 1, 2009, pp. 26-32. doi:10.4085/1062-6050-44.1.26

[8] J. Barrion, K. Crossley and I. Davis, "Gait Retraining to Reduce the Knee Adduction Moment through Real-Time Visual Feedback of Dynamic Knee Alignment," Journal of Biomechanics, Vol. 43, No. 11, 2010, pp. 2208-2213. doi:10.1016/i.jbiomech.2010.03.040

[9] J. Barrios, J. Higginson, T. Royer and I. Davis, "Statistics and Dynamic Correlates of the Knee Adduction Moment in Healthy Knees Ranging from Normal to Varus-Aligned," Clinical Biomachanics, Vol. 24, No. 10, 2009, pp. 850-854. doi:10.1016/j.clinbiomech.2009.07.016

[10] K. Tallroth, A. Harilainen, L. Kerttula and R. Sayed, “Ankle, Osteoarthritis Is Associated with Knee Osteoarthritis. Conclusions Based on Mechanical Axis Radiographics," Archives of Orthopaedic and Trauma Surgery, Vol. 128, No. 6, 2008, pp. 555-560. doi:10.1007/s00402-007-0502-9

[11] J. Bellemans, W. Colyn, H. Vandenneucker and J. Victor, "Is Neutral Mechanical Alignment Normal for All Patients? The Concept of Constitutional Varus," The Association of Bone and Joint Surgeons, Rosemont, 2001.

[12] E. Witvrouw, L. Danneels, Y. Thijs, D. Cambier and J. Bellemans, "Does Soccor Participation Lead to Genu Varum?" Knee Surgery, Sports Traumatology, Arthroscopy, Vol. 17, No. 4, 2009, pp. 422-427. doi:10.1007/s00167-008-0710-z

[13] A. Colebatch, D. Hart, G. Zhai, F. Williams, T. Spector and N. Arden, "Effective Measurement of Knee Alignment Using AP Knee Radiographs," The Knee, Vol. 16, No. 1, 2009, pp. 42-45. doi:10.1016/i.knee.2008.07.007

[14] J. R. Moreland, L. W. Bassett and G. J. Hanker, "Radiographic Analysis of the Axial Alignment of the Lower Extremity," The Journal of Bone \& Joint Surgery, Vol. 69A, No. 5, 1987, pp. 745-749.

[15] J. Sailer, M. Scharitzer, P. Peloschek, et al., "Quantification of Axial Alignment of the Lower Extremity on Conventional and Digital Total Leg Radiographs," European Journal of Radiology, Vol. 15, No. 1, 2005, pp. 170-173. doi:10.1007/s00330-004-2436-8

[16] S. Sabharwal, C. Zhao and M. Edgar, "Lower Limb Ali- gnment in Children: Reference Values Based on a FullLength Standing Radiograph," Journal of Pediatric Orthopaedics, Vol. 28, No. 7, 2008, pp. 740-746. doi:10.1097/BPO.0b013e318186eb79

[17] J. C. Cheng, P. S. Chan, S. C. Chaing and P. W. Hui, "Angular and Rotational Profile of the Lower Limb in 2630 Chinese Children," Journal of Pediatric Orthopaedics, Vol. 11, No. 2, 1991, pp. 154-161. doi:10.1097/01241398-199103000-00003

[18] M. Arazi, T. C. Ögün and R. Memik, "Normal Development of the Tibiofemoral Angle in Children: A Clinical Study of 590 Normal Subjects from 3 to 17 Years of Age," Journal of Pediatric Orthopaedics, Vol. 21, No. 2, 2001, pp. 264-267. doi:10.1097/01241398-200103000-00027

[19] D. Paley, "Priciples of Deformity Correction," SpringerVerslag, Heidelberg, 2003, pp. 1-60.

[20] E. Y. Chao, E. V. Neluheni, R. W. Hsu and D. Paley, "Biomechanics of Malalignment," Orthopedic Clinics of North America, Vol. 25, No. 3, 1994, pp. 379-386.

[21] T. D. Cooke, J. Li and R. A. Scundamore, "Radiographic Assasment of Bony Contributions to Knee Deformity," Orthopedic Clinics of North America, Vol. 25, No. 3, 1994, pp. 387-393.

[22] T. D. Cooke, A. Scundamore, J. Li, U. W. Bryant and P. Costigan, "Axial Lower-Limb Alignment: Comparison of Knee Geometry in Normal Volunteers and Osteoarthritis Patients," Osteoarthritis and Cartilage, Vol. 5, No. 1, 1997, pp. 39-47. doi:10.1016/S1063-4584(97)80030-1

[23] R. W. Hsu, S. Himeno, M. B. Coventry and E. Y. Chao, "Normal Axial Alignment of the Lower Extremity and Load-Bearing Distribution at the Knee," Clinical Orthopaedics and Related Research, Vol. 255, 1990, pp. 215227.

[24] J. Y. Jenny, C. Boeri and L. Ballonzoni, "Coronal Alignment of the Lower Extremity," Acta Orthopaedica, Vol. 76, No. 3, 2005, pp. 403-407.

[25] A. D. Nguyen and S. J. Schultz, "Sex Differences in Clinical Measures of Lower Extremity Alignment," Journal of Orthopaedic \& Sports Physical Therapy, Vol. 37, 2007, pp. 289-339.

[26] W. M. Tang, Y. H. Zhu and K. Y. Chiu, "Axial Alignment of the Lower Extremity in Chinese Adults," Journal of Bone \& Joint Surgery, Vol. 82, 2000, pp. 1603-1608.

[27] M. Shields, S. C. Gorber, I. Janssen and M. S. Tremblay, "Obesity Estimates for Children Based on Parent-Reported versus Direct Measures," Statistics Canada, Catalogue No. 82-003-XPE, Health Reports, Vol. 22, No. 3, 2011. 